\title{
Adaptive entry guidance for the Tianwen-1 mission
}

\author{
Minwen Guo ${ }^{1,2}(\bowtie)$, Xiangyu Huang ${ }^{1,2}$, Maodeng $\mathbf{L i}^{1,2}$, Jinchang $\mathbf{H u}^{1,2}$, and Chao $\mathbf{X u}^{1,2}$ \\ 1. Beijing Institute of Control Engineering, Beijing 100094, China \\ 2. National Key Laboratory of Science and Technology on Space Intelligent Control, Beijing 100094, China
}

\begin{abstract}
To meet the requirements of the Tianwen-1 mission, adaptive entry guidance for entry vehicles, with low lift-to-drag ratios, limited control authority, and large initial state bias, was presented. Typically, the entry guidance law is divided into four distinct phases: trim angle-of-attack phase, range control phase, heading alignment phase, and trim-wing deployment phase. In the range control phase, the predictor-corrector guidance algorithm is improved by planning an on-board trajectory based on the Mars Science Laboratory (MSL) entry guidance algorithm. The nominal trajectory was designed and described using a combination of the downrange value and other states, such as drag acceleration and altitude rate. For a large initial state bias, the nominal downrange value was modified onboard by weighing the landing accuracy, control authority, and parachute deployment altitude. The biggest advantage of this approach is that it allows the successful correction of altitude errors and the avoidance of control saturation. An overview of the optimal trajectory design process, including a discussion of the design of the initial flight path angle, relevant event trigger, and transition conditions between the four phases, was also presented. Finally, telemetry data analysis and post-flight assessment results were used to illustrate the adaptive guidance law, create good conditions for subsequent parachute reduction and power reduction processes, and gauge the success of the mission.
\end{abstract}

\section{KEYWORDS}

Tianwen-1 mission

adaptive entry guidance on-board trajectory planning telemetry data analysis post-flight assessment

\section{Research Article}

Received: 16 September 2021

Accepted: 25 October 2021

(C) The Author(s) 2022

\section{Introduction}

The Tianwen-1 mission flew a lifting entry by offsetting the center of mass to produce a trim angle of attack, and used the active on-board guidance to improve the landing accuracy, similar to the case in the successful MSL mission [1]. The design of the entry, descent, and landing (EDL) process using the guidance and control law is a challenging issue for the Tianwen-1 Mars mission. The EDL process starts from the atmospheric entry interface at an ellipsoid altitude of $125 \mathrm{~km}$ and an initial velocity of $17,000 \mathrm{~km} / \mathrm{h}$. During this process, the vehicle undergoes rapid deceleration through a tenuous atmosphere, while autonomously and reliably deploying the trim wing and the parachute. In this paper, the design of the Tianwen-1 adaptive entry guidance law is described.

To meet the requirements of the Tianwen- 1 mission, it is essential to balance the lift of the vehicle to minimize the range error while ensuring safe deployment. Thus, several considerations, such as selecting deploying altitudes that are as high as possible, and choosing robust deployment triggers, must be made to design in the entry guidance. The initial primary task of the entry guidance [2-4] was to increase the landing accuracy, without sufficient concern for the parachute deployment condition. Moreover, due to the limited control authority of the entry vehicle and the large initial state bias, considerable attention will be required to reduce the downrange and cross-range error, resulting in control saturation. However, the altitude error cannot be corrected in real time, resulting in the parachute deployment altitude being a few kilometers off-nominal. Further, the altitude and dynamic pressure may not meet the parachute deployment constraints, rendering a landing mission impossible. 


\section{Nomenclature}

AOA

$\mathrm{CR}$

DR

EFPA

EI angle of attack (rad)

crossrange

downrange

entry flight path angle

entry interface
GNC

IMU

$L / D$

$\beta$ guidance, navigation, and control

inertial measurement unit

current ratio of lift-to-drag

ballistic coefficient
The entry vehicle for the Tianwen-1 mission has low lift-to-drag ratio, limited control authority, and large initial state bias. This causes the parachute deployment constraints [5] that are based on the altitude and dynamic pressure to be unmet, which threaten the security of parachute deployment. To solve the aforementioned problem, the traditional nominal trajectory-based guidance law [6] cannot be adopted for a large initial state bias without the occurrence of multi-fold growth in the entry guidance gain file [7]. The analytical predictor design and the entry guidance performance of the different guidance methods were analyzed in Ref. [8]. As suggested in Ref. [8], the main advantage of predictive guidance is that online updating of the reference trajectory enables to compensate for tracking errors and to improve guidance precision. Adaptive on-board guidance for entry vehicles was investigated in Ref. [9]. Feedback linearization, an effective method for nonlinear control, was applied without considering the initial state bias to address drag profile tracking problems. In Ref. [10], an adaptive entry guidance law was designed to improve landing precision and to compensate for deviations in atmospheric density and aerodynamic coefficients. The altitude for parachute deployment is a primary consideration in guidance law design [11]. In Ref. [11], an improved altitude control algorithm was presented by adjusting the bank angle using the phase with a low Mach number.

In this study, an adaptive entry guidance logic based on on-board trajectory planning and an overview of the design process was used to generate optimized guidance tactics. Using the guidance law, the nominal trajectory is first designed and described by the downrange value and the other states, such as drag acceleration and altitude rate. Next, owing to the large initial longitude and latitude bias, we analyzed the maximum allowable range for the initial downrange, considering the flyability of the lander and the parachute deployment constraints. If the actual downrange exceeds the allowable range, the planned onboard trajectory law is adopted. The most important aspect is to modify the nominal downrange value by calculating and compensating for the deviation between the actual value and the allowable value. By using the new nominal downrange value, the successful correction of the altitude error and the avoidance of control saturation, which result from a large initial state bias, is possible using the updated guidance law. The guidance law then accurately drives the lander to the desired landing position with an altitude error as small as possible. To investigate the utility of the proposed method, a simulation was performed to allow for comparison with the MSL guidance law of Ref. [7]. Additionally, optimization designs, such as the improvement of the initial flight path angle and the relevant event trigger designs are also discussed in this study. Finally, telemetry data analysis and post-flight assessment results are shown to demonstrate the perfect performance of the adaptive guidance law for the Tianwen-1 mission.

\section{Reference trajectory design}

The entry process of Tianwen- 1 is divided into four phases: trim angle-of-attack (AOA), range control, heading alignment, and trim-wing deployment phases. The key events during the four entry guidance phases are the deployment of the trim wing and the parachute. In addition to the project requirements and design principles, it is important to design the transition conditions between the four phases, such as when to begin the range control, when to begin the heading alignment control, and when to deploy the trim wing and the parachute. These are important for reference trajectory planning and enable the definition of the design requirements and optimization of the initial entry flight path angle (EFPA).

\subsection{Relevant project requirements}

Tianwen- 1 requires a touchdown ellipse of $50 \mathrm{~km} \times 25 \mathrm{~km}$ in a flat area selection within the constraining criteria 
of the trim-wing and the parachute deployment altitude, Mach number, and dynamic pressure.

\subsubsection{Dynamic pressure}

A trim-wing deployment dynamic pressure between 500 and $1200 \mathrm{~Pa}$ is required. If the dynamic pressure is greater than $1200 \mathrm{~Pa}$, trim wing breakage will occur. If the dynamic pressure is too low, it may cause the trim-wing deployment to fail. Similarly, an adaptive dynamic pressure between 360 and $760 \mathrm{~Pa}$ is critical for chute deployment. A sufficient dynamic pressure is beneficial for ensuring inflation. The maximum value limit restrains the resulting peak inflation loads, which may cause the chute to fail.

\subsubsection{Mach number}

The Mach number for the chute deployment time should be restricted between 1.5 and 2.1. The Mach number affects the aeroheating and inflation dynamics of the chute. If the Mach number is too high, the parachute may experience excessive heating at the stagnation point and violent inflation may occur. If the Mach number is too low, the inflation dynamics may not be sufficient to deploy the chute.

\subsubsection{Deployment altitude}

A minimum parachute deployment altitude of $4 \mathrm{~km}$ is required to allow sufficient time to complete the subsequent descent and landing tasks [12-15]. Here, the altitude is referenced to the Mars ellipsoid altitude, which is approximately equal to the Mars Orbiter Laser Altimeter (MOLA) altitude. Below this altitude, the chute and propulsive system cannot decelerate the lander in time for a soft landing.

\subsection{Initial EFPA and reference bank angle design}

The reference trajectory is designed to achieve the highest deployment altitude for a given vehicle configuration and atmospheric conditions, because the selected landing site is large and the range is indeterminate. First, the entry corridor is calculated by considering the heating and the parachute deployment dynamic pressure. To increase the time for favorable communication links, the magnitude of the bank angle was limited to $0^{\circ}-90^{\circ}$. Subsequently, we carried out a simulation to acquire thousands of reference trajectories for the undispersed cases. In each simulation, the traversal bank angle records a value for each degree, and the flight path angle records a value for every $0.1^{\circ}$ within the scope of the constraint. A contour plot of the simulation results is shown in Fig. 1.

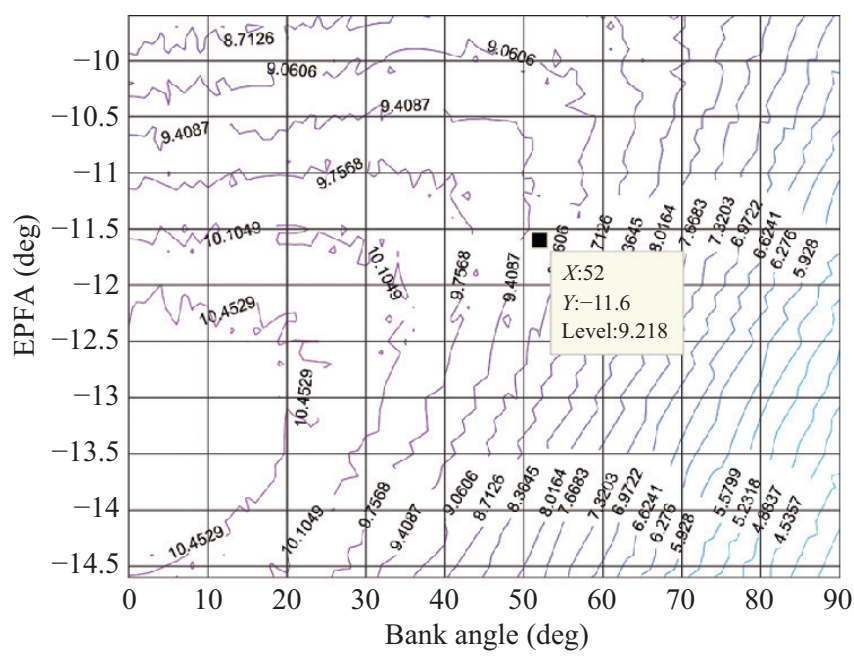

Fig. 1 Contour map showing the parachute deployment altitudes for the reference design.

In Fig. 1, one can observe that the altitude changes as a function of the entry flight path angle and the bank angle. The parachute deployment altitude was higher until a peak was reached, after which it decreased. This is due to the entry vehicle entering the atmosphere with a shallower flight path angle and then flying with a fixed bank angle, indicating that there is an optimal flight path angle to any bank angle which helps the vehicle reach the highest parachute deployment altitude. Moreover, the maximum altitude increased as the bank angle decreased.

Similarly, the downrange contour map can be plotted, after which the reference bank angle profile with a sufficient vertical current lift-to-drag $(L / D)$ ratio can be set and held in reserve to modify the range error. By considering the robustness of the range control and the optimized parachute deployment altitude, the reference trajectory was designed with a $-11.6^{\circ}$ entry flight path angle and a $52^{\circ}$ bank angle.

\subsection{Trim-wing and parachute deployment triggers}

In other successful Mars missions, the parachute deployment trigger design considered only one simple condition, such as dynamic pressure (in the Pathfinder mission) or Mach number (in the Curiosity and Perseverance missions). In fact, the key events in the EDL process, such as trim-wing and the parachute 
deployment, are interrelated and irreversible. Each action trigger needs to meet strict constraints, such as dynamic pressure, altitude, and Mach number constraints. The guidance, navigation, and control (GNC) system must be able to perform fully-autonomous decisions under a complex and uncertain Martian environment. Thus, it is crucial that event triggers are designed to be reliable and fault-tolerant. The navigation system only uses inertial measurement unit (IMU) acceleration measurements during entry, lacking other sensors to provide information on the position and velocity. If the parachute deployment trigger relies only on navigated state estimates, such as the Mach number or the dynamic pressure, which are greatly affected by the accuracy of the initial orbit and attitude estimation as well as the error of the inertial sensor, it is probable that some other constraints cannot be met when the event is triggered; thus, the safety of the landing scenario will be seriously affected. In view of the above problems, a highly fault-tolerant triggering method is presented in this paper for critical events in the EDL process of Mars exploration in China. In the design, a master and backup triggering scheme was adopted to improve reliability. The following is an example of a trigger condition design for parachute deployment.

\subsubsection{Main parachute deployment trigger design}

In general, we chose the Mach number or dynamic pressure as the main judgment condition. The judgment should be made according to the flight ability of the actual object, depending on which is the more proper condition. The flight capability referred to here is related to the aerodynamic characteristics of the entry vehicle and the Martian atmospheric environment parameters (atmospheric density and temperature). Specifically, it is important to understand the capability of the relationship curve between the Mach number and the dynamic pressure near the parachute deployment of the flight trajectory, as shown in Fig. 2. From the actual flight trajectory, we selected the main parachute deployment trigger using a constraint condition that is not easily satisfied and is regarded as a more stringent condition. The red lines in Fig. 2 represent the constraint ranges of the Mach number and dynamic pressure. When the Mach number exceeds the constraint (greater than 2.3 Mach), the dynamic pressure still meets the constraint; therefore, the Mach number was chosen as the primary trigger condition.
Furthermore, 1000 Monte Carlo dispersed simulations were conducted to verify the aforementioned results with aerodynamic model deviation, atmospheric density, temperature, and other state parameter deviations of the entry interface (EI). According to the performance results shown in Fig. 3, the pressure exceeds the boundary much earlier than the Mach number only once, and thus it is more reasonable to choose the Mach number as the trigger. Additionally, we can see that dynamic pressure dispersion corresponding to Mach number 1.8 is the smallest. Therefore, the triggering condition for parachute deployment is that the Mach number is greater than 1.8 .

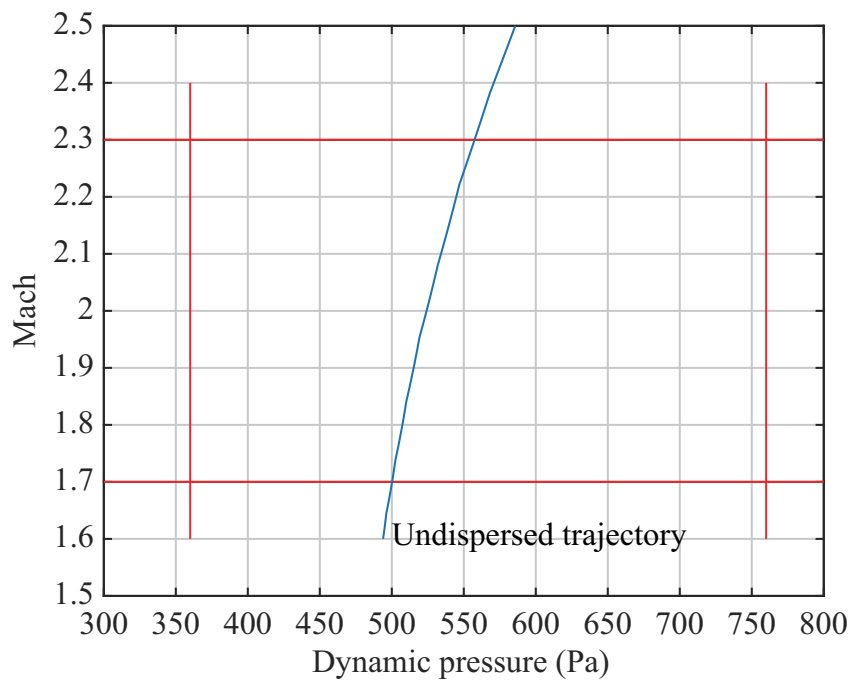

Fig. 2 Mach number vs. dynamic pressure of an undispersed trajectory.

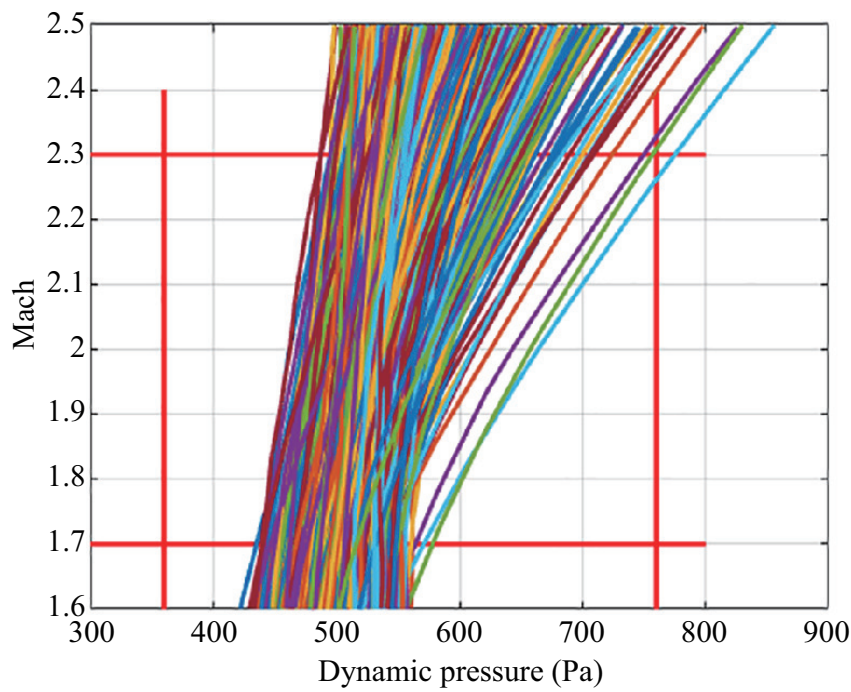

Fig. 3 Mach number vs. dynamic pressure of the dispersed trajectories. 


\subsubsection{Backup parachute deployment trigger design}

The design of the backup parachute deployment trigger is discussed below. The cumulative value of the axial apparent velocity increment, which can be directly measured by an accelerometer, was selected as the backup trigger.

Numerous dispersed simulations were performed, and the cumulative value of the axial apparent velocity increment was determined. The cumulative values from the EI to the trim-wing opening, when the main Mach number condition (2.8 Mach) was triggered, and from the EI to the parachute opening (1.8 Mach) were calculated. According to the statistical results, the maximum value of the cumulative axial apparent velocity increment was selected as the backup trigger condition. Next, the feasibility of the backup trigger must be verified by a Monte Carlo simulation. In the simulation, only the backup trigger was used as the judgment condition. Subsequently, we verified whether all the constraints at the trim-wing and the parachute deployment were met.

\subsection{Heading alignment fixed velocity trigger}

The critical velocity $V_{\mathrm{t}}$, which is the velocity of transition from the range control to the heading alignment, can be determined using the following steps. First, the dynamic model is simplified. The centrifugal and gravitational forces are relatively small in comparison with the aerodynamic forces during the process of re-entry. Therefore, these two forces can be neglected. Consider the Mars-relative longitudinal motion state of a vehicle. The simplified equation of motion can be described by

$$
\left\{\begin{array}{l}
\dot{r}=v \sin \gamma \\
\dot{s}=v \cos \gamma \\
\dot{V}=-D-g \sin \gamma \\
\dot{\gamma}=\frac{1}{V}\left[L \cos \sigma+\left(\frac{V^{2}}{r}-g\right) \cos \gamma\right]
\end{array}\right.
$$

According to the formula $V \dot{\gamma}=L \cos \sigma+\left(\frac{V^{2}}{r}-\frac{\mu}{r^{2}}\right)$ - $\cos \gamma$, letting $\dot{\gamma}=0$, a velocity profile can be obtained as Eq. (2):

$$
V=\sqrt{\frac{\mu / r^{2}}{0.5 \times \rho / \beta \times L / D \times \cos \left(\sigma^{*}\right)+1 / r}}
$$

where $g$ is the Martian gravity constant. Another velocity profile is the reference trajectory. The velocity at the intersection point of the two velocity profiles is the critical velocity, as shown in Fig. 4.

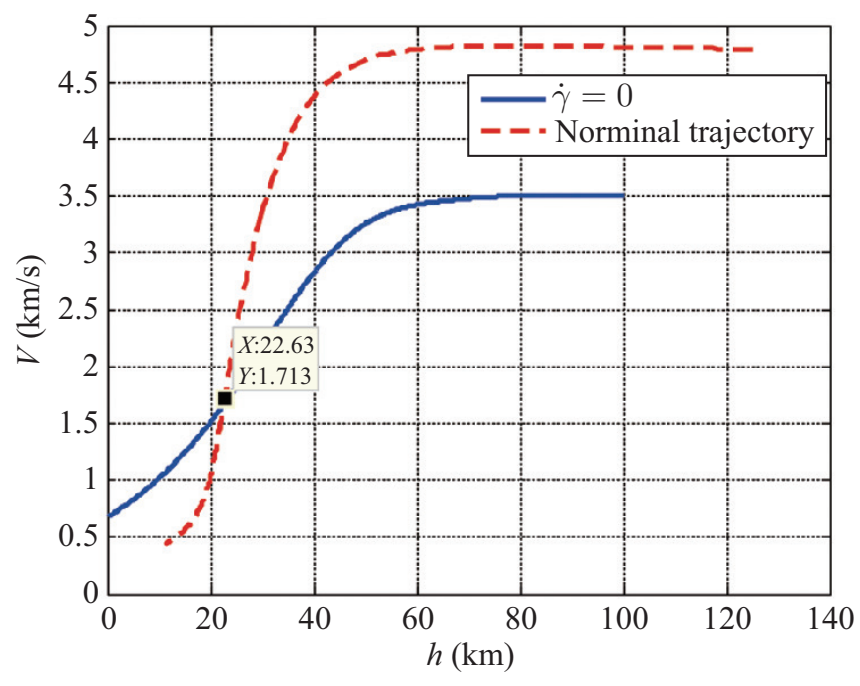

Fig. 4 Relative velocity vs. altitude of nominal trajectory and $\dot{\gamma}=0$ trajectory.

\section{Adaptive entry guidance}

In this section, a guidance law was designed using online trajectory planning. First, an overview of the guidance law is presented. Next, an adaptive online trajectory design of the guidance law was developed.

\subsection{Guidance law overview}

The entry guidance is divided into four distinct phases (as shown in Fig. 5) as discussed below in the order of their occurrence.

(1) The trim angle-of-attack phase. In this phase, the drag acceleration magnitude is smaller than $0.2 g$, where $g$ is the Earth's gravity constant. Owing to the small aerodynamic force, the downrange control is not sensitive to the different bank angle commands. The bank angle command is constant at this phase associated with the initial nominal bank angle, and the angle of attack is maintained at the expected trim angle. At the beginning of this phase, the adaptive range compensation and online trajectory planning algorithm, which will be discussed in Section 4.2, was conducted if the initial longitude and latitude error were greater than the threshold.

(2) Range control phase. Once the drag acceleration magnitude exceeds $0.2 g$, the GNC flight software of the lander begins range control using an analytical predictor-corrector guidance algorithm. During this phase, the entry guidance law predicts the downrange to go to error based on the deviation of drag and altitude rate with respect to the nominal reference trajectory profile. Then, the bank angle command is generated to 


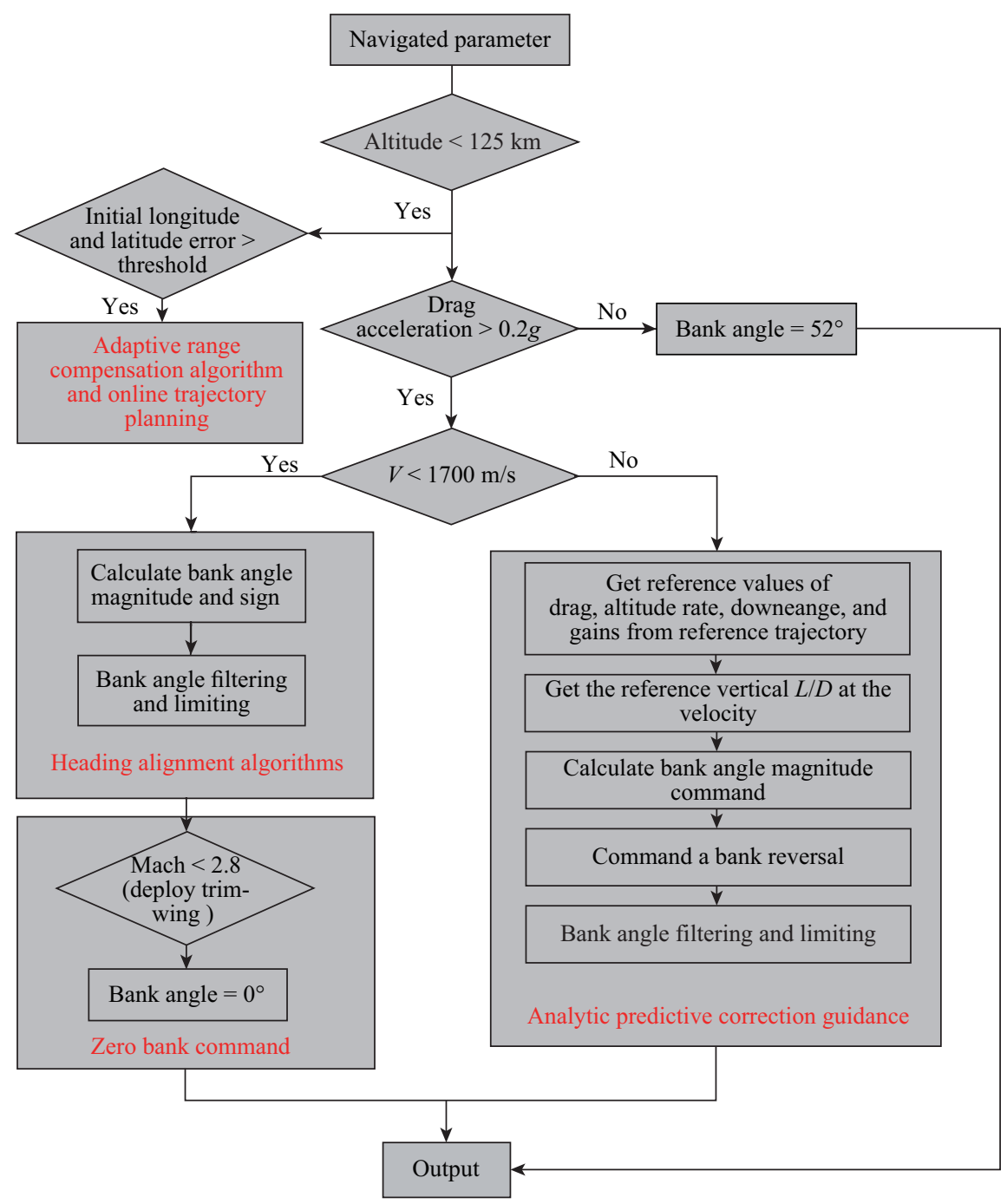

Fig. 5 Flow chart for entry guidance.

correct for range errors, as shown in Eq. (3):

$$
\begin{aligned}
& (L / D) \cos \sigma=(L / D)^{*} \cos \sigma^{*}+\frac{K}{F_{3}(V)}\left[-\left(\mathrm{DR}-\mathrm{DR}^{*}\right)-\right. \\
& \left.F_{2}(V)\left(\dot{h}-\dot{h}^{*}(V)\right)-F_{1}(V)\left(D-D^{*}(V)\right)\right]
\end{aligned}
$$

where $h$ is the altitude, $h$ is the altitude rate, DR is the downrange, $V$ is the relative velocity, and $\sigma$ is the bank angle. The reference value is represented by the superscript $*$, the feedback coefficients are $F_{1}, F_{2}$, and $F_{3}$, and $K$ is set to a value of 5 to produce an "over control" condition $[16,17]$. The predicted cross-range on landing was used in the controller to correct the cross-range error.

(3) Heading alignment phase. Once the velocity has dropped past a critical value $V_{\mathrm{t}}$, the guidance ceases range control and begins heading alignment. When the velocity decreases, the lift force restricts the rate of the flight path angle to be greater than zero. Therefore, the bank angle is not effective for commanding the control range. Then, the bank angle is commanded to steer the lander to offset the cross-range error.

The bank angle command can be obtained as Eq. (4):

$$
\left\{\begin{array}{l}
\sigma=K_{1} \arctan \left(\frac{\mathrm{CR}_{\text {pre }}}{S_{\text {togo }}}\right) \\
S_{\text {togo }}=S_{\text {total }}-\mathrm{DR} \\
|\sigma|<15^{\circ}
\end{array}\right.
$$

where $K_{1}$ is set to a value of ten to produce an "over control" condition, $\mathrm{CR}_{\text {pre }}$ is the predicted crossrange on landing, which is different from the MSL entry guidance algorithm, and $S_{\text {togo }}$ is the downrange to go to the desired landing point.

(4) Trim-wing deployment phase. The bank command is modulated to $0^{\circ}$ when the trim wing is deployed. 


\subsection{Adaptive guidance law}

First, the allowed maximum initial downrange $\left[\mathrm{DR}_{\min -}\right.$, $\mathrm{DR}_{\max +}$ ] should be analyzed using a Monte Carlo simulation, considering all model parameter uncertainties, bank angle constraints, and all parachute deployment constraints. Then, the actual initial downrange $\mathrm{DR}_{0}$ is calculated using the longitude and latitude of the actual initial entry point and target landing point. The deviation of the initial downrange and the maximum scope is then given by $\mathrm{DR}_{\text {err }}=\mathrm{DR}_{0}-\mathrm{DR}_{\text {min- }-/ \max +}$, where $\mathrm{DR}_{\text {err }}$ is the compensation of the initial downrange error in different cases. Once the initial longitude and latitude bias are larger than the threshold value, the reference total downrange to go should be updated as $\mathrm{DR}_{\text {total }}^{\text {new } *}=\mathrm{DR}_{\text {total }}^{*}-\mathrm{DR}_{\text {err }}$. Then, the trajectory should be redesigned online using the Newton iteration method:

$$
x_{n+1}=x_{n}-\frac{x_{n}-x_{n-1}}{f\left(x_{n}\right)-f\left(x_{n-1}\right)} f\left(x_{n}\right)
$$

where $x=\cos \sigma^{*}$ and $f(x)=\mathrm{DR}_{\text {total }}^{\text {new }}-\operatorname{DR}_{\text {total }}\left(\sigma^{*}\right)$ are the numerically predicted downrange errors between the predicted total downrange $\operatorname{DR}\left(\sigma^{*}\right)$ with an online estimated drag acceleration and $L / D$, and the reference total downrange to go. Finally, we substitute the updated downrange reference value with $\mathrm{DR}_{\text {new }}^{*}$ in Eq. (3) to produce the following adaptive guidance law:

$$
\begin{aligned}
& (L / D) \cos \sigma=(L / D)^{*} \cos \sigma^{*}+\frac{K}{F_{3}(V)}\left[-\left(\mathrm{DR}-\mathrm{DR}_{\text {new }}^{*}\right)\right. \\
& \left.-F_{2}(V)\left(\dot{h}-\dot{h}^{*}(V)\right)-F_{1}(V)\left(D-D^{*}(V)\right)\right]
\end{aligned}
$$

To demonstrate the effectiveness of the proposed

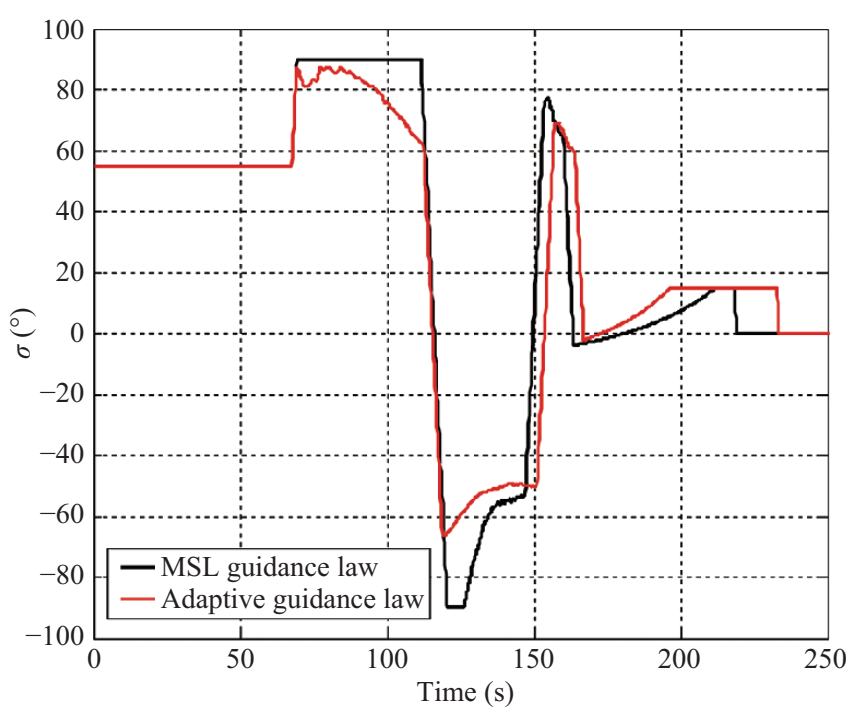

Fig. 6 Guidance law command comparison. guidance law, a numerical simulation was conducted using the parameters of the MSL entry vehicle. Figures 6 and 7 illustrate the performance comparison when the initial latitude error is equal to $0.4^{\circ}$ with the application of the presented guidance law and MSL guidance law. The control saturation problem that occurred in the initial entry phase when using the MSL guidance law is shown in Fig. 6. The executed bank angle was $90^{\circ}$, which lasted for $50 \mathrm{~s}$. The proposed adaptive guidance law avoids control saturation by adopting the onboard trajectory-planned algorithm. Moreover, as shown by the subgraph in Fig. 7, the parachute altitude is found to be $10.78 \mathrm{~km}$ using the MSL guidance law, while $11.29 \mathrm{~km}$ is obtained when the proposed law is applied. The presented law provides a higher parachute altitude of approximately $500 \mathrm{~m}$.

\section{Telemetry data analysis and post-flight assessment}

In this section, the actual flight results for the Tianwen-1 EDL process are presented. For the 537-s EDL process, the lander landed successfully on Mars. The actual latitude and longitude for the landing site and the navigated landing site are $25.066^{\circ} \mathrm{N}, 109.925^{\circ} \mathrm{E}$ and $25.09^{\circ} \mathrm{N}, 109.9^{\circ} \mathrm{E}$, respectively; the actual downrange and cross-range errors with respect to the pre-defined landing sites $\left(25.1188^{\circ} \mathrm{N}, 109.9305^{\circ} \mathrm{E}\right)$ are 3.1 and $0.2 \mathrm{~km}$, respectively.

The navigated entry trajectories collected from telemetry are shown in Figs. 8-11. The entry interface

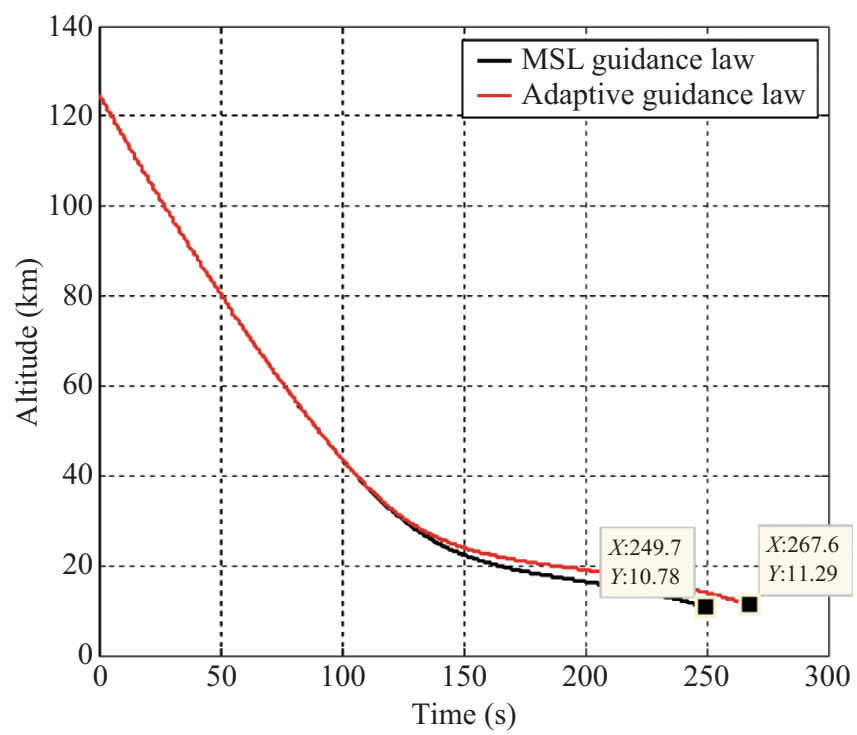

Fig. 7 Altitude trajectory comparison.

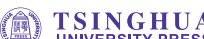
Springer 
arrives at a bank angle of $0^{\circ}$, an angle of attack of $-10^{\circ}$, and a sideslip angle of $0^{\circ}$ at a planet-relative velocity of $4.9 \mathrm{~km} / \mathrm{s}$ and $25 \mathrm{Mach}$. The time from entry to parachute deployment typically lasts $279 \mathrm{~s}$, in which the AOA-trim mode takes $68 \mathrm{~s}$. When the sensed acceleration magnitude of the descent module exceeded $1.96 \mathrm{~m} / \mathrm{s}^{2}$ at a navigated altitude of approximately $63 \mathrm{~km}$ and a navigated velocity of approximately $24 \mathrm{Mach}$, the lift range control phase began. The heading alignment began at a navigated velocity of $8.1 \mathrm{Mach}$, and the trim wing was deployed at 2.8 Mach, after which the trim values of the total angle of attack approached approximately zero from nearly $10^{\circ}$. The time of the trim-wing deployment was approximately $243 \mathrm{~s}$. At that instant, the altitude is approximately $19.2 \mathrm{~km}$. The parachute was deployed at a navigated velocity of 1.8 Mach when the actual altitude was approximately $13 \mathrm{~km}$.

In Fig. 8, the $L / D$ ratio estimation of the entry vehicle changes with the trim angle of attack in the entire process. The maximum dynamic pressure was below $4000 \mathrm{~Pa}$, and the dynamic pressure of the trim-wing deployment and the parachute deployment both met the constraints. In Fig. 9, we can see that there are four bank reversals for the entire entry process. After the control target transitioned to heading alignment from range control, the bank angle was limited to $15^{\circ}$ until the trim wing was deployed. This limiter improves the parachute altitude to $13 \mathrm{~km}$. However, this may lead to a derived adverse effect on cross-range error modification. After the trim wing was opened, the bank angle was kept at zero degrees. The $L / D$ ratio estimation of the entry vehicle changes with the trim angle of attack during the process. After the vehicle enters the Martian atmosphere, lift control begins.

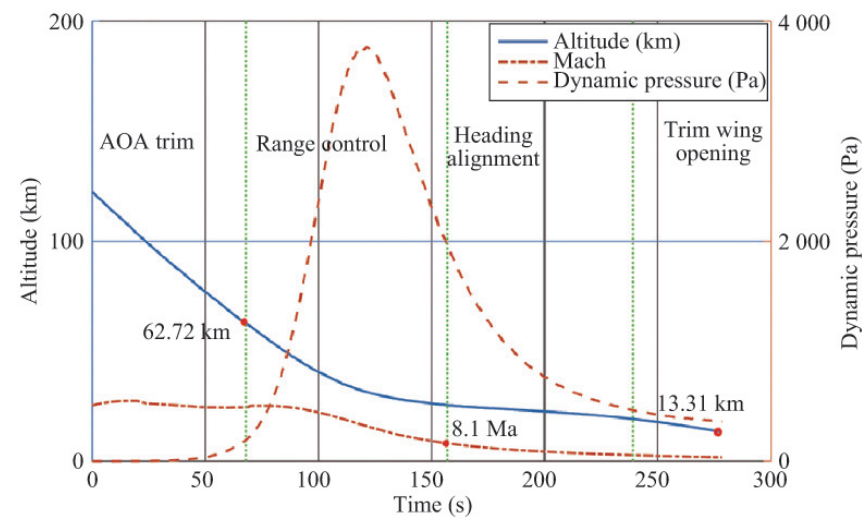

Fig. 8 Altitude, Mach number, and dynamic pressure curves.
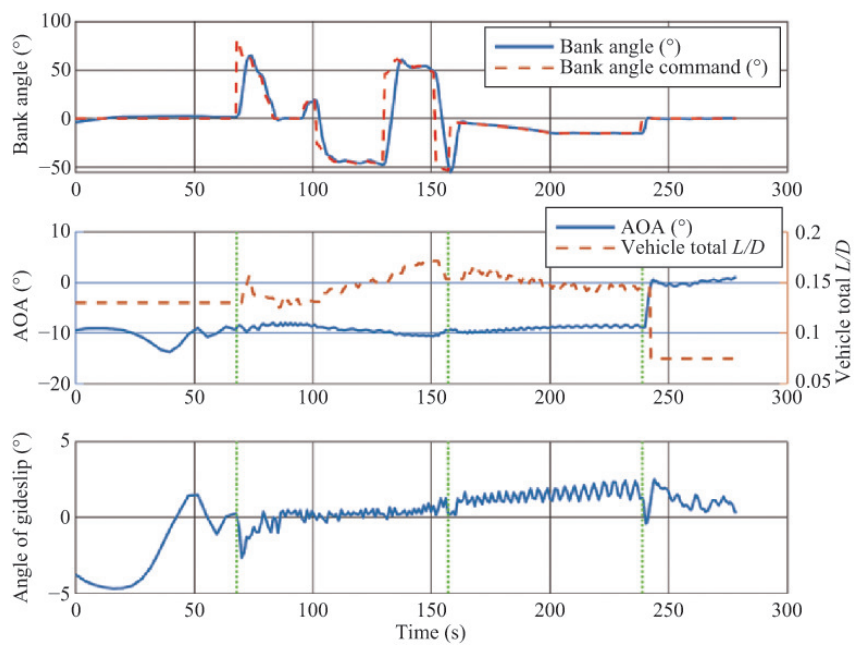

Fig. 9 Bank angle, angle of attack, angle of sideslip, and vehicle total $L / D$ curves.
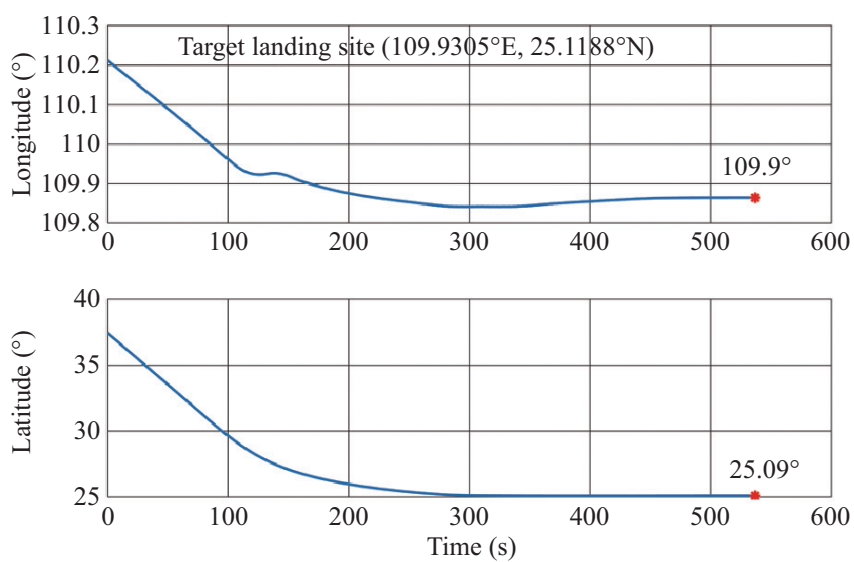

Fig. 10 Latitude and longitude curves.

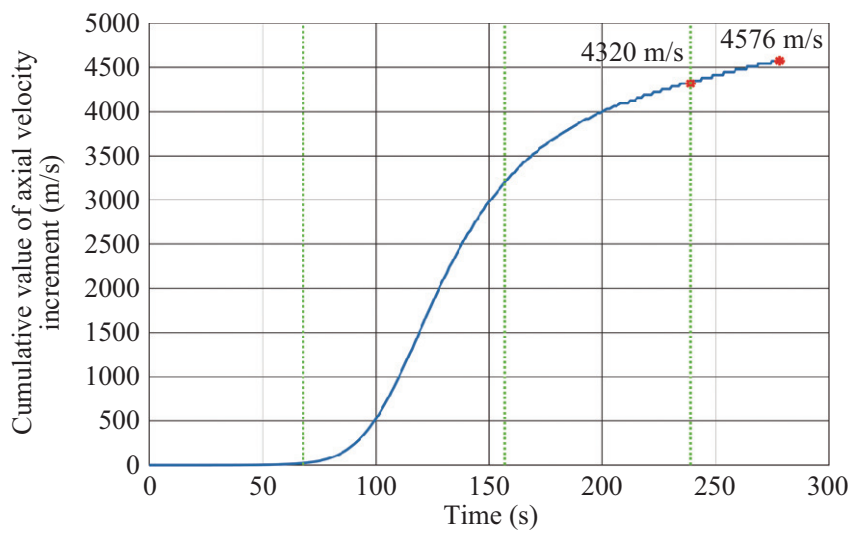

Fig. 11 Cumulative value for the axial apparent velocity increment.

Then, the angle of the sideslip was kept in the $\pm 5^{\circ}$ range, as expected. In Fig. 10, the longitude and latitude of the navigated position information changed with time and became increasingly closer to the target landing site.

\section{(10) UNIVERITY TREAS}


In Fig. 11, the cumulative values of the axial velocity increment at the trim-wing and the parachute deployment instants were 4320 and $4576 \mathrm{~m} / \mathrm{s}$, respectively, and neither violated the backup triggers.

\section{Conclusions}

A new adaptive entry guidance law that includes four phase algorithms, namely, the adaptive range compensation algorithm, analytic predictive correction guidance algorithm, heading alignment algorithm, and zero bank command algorithm, was designed for the Tianwen-1 Mars atmospheric entry process. According to the results of the control ability and the large initial state bias, the guidance law allows for the successful correction of the altitude error and avoidance of control saturation by modifying the onboard nominal trajectory. The numerous modifications for this process include initial flight path angle optimization to obtain the highest parachute deployment altitude, and a backup parachute deployment trigger to improve mission reliability. The effectiveness of the entry guidance algorithm was demonstrated using a parachute deployment altitude of approximately $13 \mathrm{~km}$, which provided plenty of time to accomplish the subsequent phase deceleration, and enabled the successful landing of Tianwen-1 with a small touchdown ellipse of $3.1 \mathrm{~km} \times 0.2 \mathrm{~km}$ at the selected landing site.

\section{Conflict of interest}

The authors declare that there is no conflict of interest.

\section{References}

[1] Mendeck, G. F., Craig, L. E. Entry guidance for the 2011 Mars Science Laboratory mission. In: Proceedings of the AIAA Atmospheric Flight Mechanics Conference, 2011: AIAA 2011-6639.

[2] Shui, Z., Zhou, J., Ge, Z. On-line predictor-corrector reentry guidance law based on Guass pseudospectral method. Journal of Astronautics, 2011, 32(6): 42-46.

[3] Tu, K. Y., Munir, M. S., Mease, K. D., Bayard, D. S. Drag-based predictive tracking guidance for Mars precision landing. Journal of Guidance, Control, and Dynamics, 2000, 23(4): 620-628.

[4] Hu, J. Adaptive predictive guidance: a unified guidance method. Aerospace Control and Application, 2019, 45(4): 53-63. (in Chinese)
[5] Guo, M., Li, M., Huang, X., Wang, D. On guidance algorithm for Martian atmospheric entry in nonconforming terminal constraints. Journal of Deep Space Exploration, 2017, 4(2): 184-189. (in Chinese)

[6] Leavitt, J. A., Mease, K. D. Feasible trajectory generation for atmospheric entry guidance. Journal of Guidance, Control and Dynamics, 2007, 30(2): 473-481.

[7] Way, D. W., Powell, R. W., Chen, A., Steltzner, A. D., Martin, A. M. S., Burkhart, P. D., Mendeck, G. F. Mars Science Laboratory: Entry, descent, and landing system performance. In: Proceedings of the IEEE Aerospace Conference, 2006.

[8] Kluever, C. A. Entry guidance performance for Mars precision landing. Journal of Guidance, Control, and Dynamics, 2008, 31(6): 1537-1544.

[9] Roenneke, A. Adaptive on-board guidance for entry vehicles. In: Proceedings of the AIAA Guidance, Navigation, and Control Conference and Exhibit, 2001: AIAA 2001-4048.

[10] Luo, T., Hu, J. Adaptive control method based on standard ballistic guidance law. Aerospace Control and Application, 2014, 40(2): 42-46. (in Chinese)

[11] Benito, J., Mease, K. D. Mars entry guidance with improved altitude control. In: Proceedings of the AIAA/AAS Astrodynamics Specialist Conference and Exhibit, 2006: AIAA 2006-6674.

[12] Zhao, H. Dynamics and Guidance for Reentry Vehicles. Changsha: Press of National University of Defense Technology, 1997. (in Chinese)

[13] Cruz, J. R., Way, D., Shidner, J., Davis, J. L., Powell, R. W., Kipp, D., Adams, D. S., Sengupta, A., Witkowski, A., Kandis, M. Parachute models used in the Mars science laboratory entry, descent, and landing simulation. In: Proceedings of the AIAA Aerodynamic Decelerator Systems Conference, 2013: AIAA 2013-1276.

[14] Li, M., Huang, X., Wang, D., Xu, C., Guo, M., $\mathrm{Hu}$, J., Zhang, X. Radar-updated inertial landing navigation with online initialization. IEEE Transactions on Aerospace and Electronic Systems, 2020, 56(5): 3360-3374.

[15] Zhang, H., Guan, Y., Huang, X., Li, J., Zhao, Y., Yu, P., Zhang, X., Yang, W., Liang, J., Wang, D. Guidance navigation and control for Chang'E-3 powered descent. Scientia Sinica Technologica, 2014, 44(4): 377-384. (in Chinese)

[16] Carman, G. L., Ives, D. G., Geller, D. K. Apollo-derived Mars precision lander guidance. In: Proceedings of the AIAA Atmospheric Flight Mechanics Conference and Exhibit, 1998: 98-4570.

[17] Mendeck, G., Carman, G. Guidance design for Mars smart landers using the entry terminal point controller. 
AIAA Atmospheric Flight Mechanics Conference and Exhibit, 2002: AIAA 2002-4502.

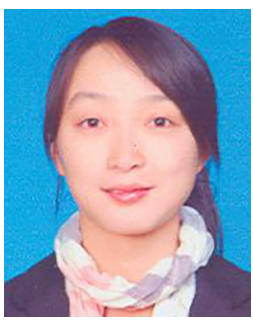

Minwen Guo received her Ph.D. degree in guidance, navigation, and control from China Academy of Space Technology in 2014. Then, she worked as an engineer at Beijing Institute of Control Engineering. From 2016 to 2021, she participated in the Mars Tianwen-1 development and was engaged in the design of the Martian atmosphere entry guidance law. In 2019, she was certified as a senior engineer. Currently, her research interests are spacecraft entry guidance and trajectory optimization. E-mail: mwguo8500@163.com.

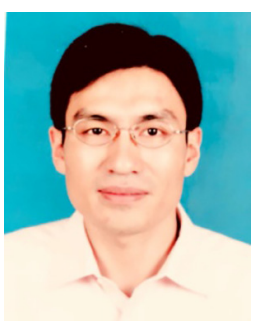

Xiangyu Huang received his B.S. and Ph.D. degrees in aerospace engineering from Harbin Institute of Technology, China, in 1999 and 2005, respectively. Since August 2005, he has been with Science and Technology on Space Intelligent Control Laboratory of Beijing Institute of Control Engineering (BICE), where he is currently a professor and senior research specialist. He was a guidance, navigation, and control (GNC) engineer of the Chang'e-3 lunar landing mission, and his current research area is GNC design for planetary landing missions. He won one State Technological Invention Award (second prize) and three Ministerial and Provincial-level Science and Technology Awards (first class). E-mail: huangxyhit@sina.com.

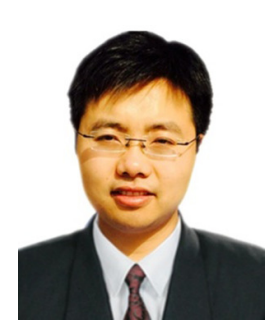

Maodeng Li received his B.S. and Ph.D. degrees in aerospace engineering from Harbin Institute of Technology, China, in 2006 and 2011, respectively. From September 2011 to August 2013, he was a postdoctoral research associate in BICE. Since August 2013, he has been with Science and Technology on Space Intelligent Control Laboratory of BICE as a senior engineer. His current research areas include spacecraft autonomous navigation and GNC design for planetary landing missions. E-mail: mdeng1985@gmail.com.

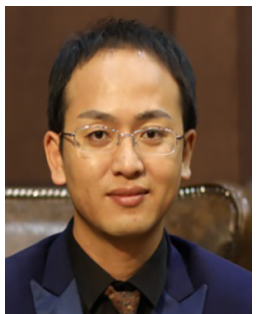

Jinchang $\mathbf{H u}$ received his B.S., M.E., and Ph.D. degrees from Beihang University, Tsinghua University, and Beijing Institute of Control Engineering, respectively. $\mathrm{He}$ is currently a senior engineer at Beijing Institute of Control Engineering. His main research interests include spacecraft attitude and orbit control. E-mail: hujinchang@tsinghua.org.cn.

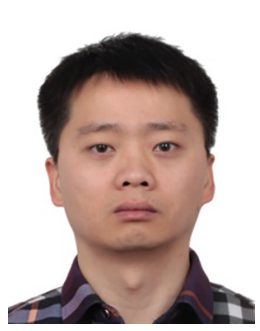

Chao Xu received his B.S. degree from Beihang University, China, in 2010 and then received his M.E. and Ph.D. degrees in guidance, navigation, and control from China Academy of Space Technology (CAST), China, in 2013 and 2017 , respectively. He is currently working as an engineer at Beijing Institute of Control Engineering (BICE), China. His research interests include autonomous navigation, vision-aided navigation, and simultaneous localization and mapping. E-mail: xc_1987@126.com.

Open Access This article is licensed under a Creative Commons Attribution 4.0 International License, which permits use, sharing, adaptation, distribution and reproduction in any medium or format, as long as you give appropriate credit to the original author(s) and the source, provide a link to the Creative Commons licence, and indicate if changes were made.

The images or other third party material in this article are included in the article's Creative Commons licence, unless indicated otherwise in a credit line to the material. If material is not included in the article's Creative Commons licence and your intended use is not permitted by statutory regulation or exceeds the permitted use, you will need to obtain permission directly from the copyright holder.

To view a copy of this licence, visit http://creativecomm ons.org/licenses/by/4.0/. 